\title{
Cognitive Dysfunction in Multiple Sclerosis: Methods and Prevalence from the GEDMA Study
}

\author{
J. Olazarán ${ }^{\mathrm{a}, \mathrm{b}} \quad$ I. Cruz ${ }^{\mathrm{b}} \quad$ J. Benito-León ${ }^{\mathrm{c}} \quad$ J.M. Morales ${ }^{\mathrm{d}} \quad$ P. Duque \\ J. Rivera-Navarro ${ }^{f}$
}

${ }^{a}$ Neurology Clinic, Hermanos Sangro Specialist Medical Centre, ${ }^{b}$ Neurology Department, Gregorio Marañón General University Teaching Hospital, ' Neurology Department, 12 de Octubre University Teaching Hospital, and ${ }^{\mathrm{d}}$ Research Department, Ministry of Labour and Social Affairs, Madrid, ${ }^{e}$ Research Unit, Virgen Macarena Hospital,

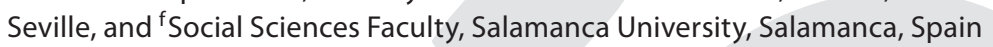

\section{Key Words}

Cognitive function screening $\cdot$ Multiple sclerosis

\begin{abstract}
Background/Aims: Cognitive dysfunction is a major handicap in multiple sclerosis (MS). Its prevalence varies due to disease heterogeneity and methodological issues. A neuropsychological battery of intermediate size was designed for and explored in the screening of cognitive dysfunction in MS patients. Methods: The battery was administered to a hospital-based sample of 191 MS patients and 50 matched controls. Eleven test scores measuring verbal fluency, verbal learning, attention, calculation and visuoperceptual ability were selected on the basis of sensitivity and lack of redundancy. Two alternative approaches were compared for diagnosis of cognitive dysfunction based, firstly, on the number of failed tasks, and secondly, on a single standardized global score. Results: The approach based on the number of failed tasks discriminated better than did the global approach between patients and controls. Using a cutoff of two altered scores, a cognitive dysfunction prevalence of $34 \%$ was obtained. The score yielded after summing errors in all tests was the most frequently altered and proved particularly useful for detecting minimally impaired patients. Conclu-
\end{abstract}

\section{KARGER \\ Fax +41 613061234 E-Mail karger@karger.ch} www.karger.com
(C) 2008 S. Karger AG, Basel

0014-3022/08/0000-0000\$24.50/0

Accessible online at:

www.karger.com/ene sion: The purpose-designed battery was adequate for the screening of cognitive dysfunction in MS patients. The better accuracy of the single-task approach might reflect MS heterogeneity.

Copyright $\odot 2008$ S. Karger AG, Basel

\section{Introduction}

As a central nervous system disease, multiple sclerosis (MS) is often accompanied by deficits in processing speed, attention, recent memory and other cognitive domains [1]. Cognitive dysfunction in MS is associated with unemployment $[2,3]$ and constitutes a major focus of interest for both diagnostic and treatment purposes [4]. However, cognitive performance displays a considerable level of variability, and there is some discrepancy about prevalence and methodology of assessment.

Cognitive evaluation should be conditioned not only by the nature of the underlying process, but also by the purpose of assessment. Comprehensive neuropsycholog-

GEDMA = Grupo de Enfermedades Desmielinizantes de Madrid (Madrid Demyelinating Disease Group).

Dr. Javier Olazarán

CEP Hermanos Sangro

Avda. de Peña Prieta, 4

ES-28038 Madrid (Spain)

Tel. +34 917306 051, Fax +34914336 033, E-Mail javier@mariawolff.es 
ical batteries are sensitive, but not affordable for screening purposes. Moreover, extensive batteries may not be suitable for severely impaired or poorly educated patients. On the other hand, short mental-status tests lack sensitivity $[5,6]$ and fail to offer a minimally detailed picture of the altered functions [7].

Intermediate-length batteries have emerged as a reasonable compromise between sensitivity and cost, and are increasingly used in both clinical and research contexts $[1,6-9]$. Nevertheless, the way in which the diagnostic value of these batteries can be optimized has not received sufficient attention. Traditionally, the prevalence and severity of cognitive dysfunction have been established on the basis of the number of altered test scores [1, $6,8]$. Alternatively, if a unitary attention and processing speed failure in MS is assumed, a single composite score might be more appropriate $[10,11]$.

We developed an intermediate-length neuropsychological battery focusing on the most frequently altered cognitive functions in MS. Sensitivity, briefness and ease of administration were prioritized. The battery was administered to a hospital-based MS cohort and matched control subjects. In order to optimize diagnostic efficiency, several cognitive scores were explored and two alternative diagnostic approaches (multiple scores vs. single composite score) were compared.

\section{Patients and Methods}

\section{Patients}

The study population consisted of 191 MS patients randomly selected for the second wave of the Madrid Demyelinating Disease Group (Grupo de Enfermedades Desmielinizantes de Madrid, GEDMA) study, a survey of physical, neuropsychological and social issues of MS in a cohort of 371 patients recruited from 13 hospitals in Madrid. MS patients were recruited by random sampling of MS databases at each hospital. Demographic and clinical characteristics of the inception cohort were similar to those described in population-based MS studies. The GEDMA study began in 2000 and the cohort was followed up over 2 years. Fuller details of the sample design and eligibility criteria have been published elsewhere [12]. Briefly, a senior neurologist with expertise in MS (J.B.-L.) reviewed patient charts to determine clinical characteristics and eligibility. All patients met criteria for clinically confirmed MS [13] and were free from any MS exacerbation at the time of assessment. Disease duration was defined as the period between symptom onset and assessment date. Neurological impairment and disability were rated according to the Expanded Disability Status Scale (EDSS) [14]. Current medications were also recorded.

The second wave of the GEDMA study was implemented in two stages: in the first of these, a quality of life instrument [15], a brief screening tool for cognitive performance [16] and a fatigue scale were administered [17]; in the second stage, subjects were visited by a trained neuropsychologist who performed formal neuropsychological (see the neuropsychological battery below) and psychiatric assessments. Neuropsychologists were blind to clinical charts and to first-step measurements. They established dementia and psychiatric diagnosis in accordance with the Diagnostic and Statistical Manual of Mental Disorders (ed. 4, DSMIV) criteria [18]. All patients gave their written informed consent before assessment, and the Móstoles General Hospital Ethics Committee approved of all aspects of the study.

\section{Control Subjects}

Fifty subjects were matched to patients by age, sex and education. Controls were chosen from among researchers' acquaintances and health workers. Subjects in the control group reported no relevant cognitive complaints, suffered from no psychiatric illnesses and had no systemic or neurological conditions that could affect cognition.

\section{Neuropsychological Battery}

The following cognitive tests were chosen on the basis of expected patterns $[1,2]$, personal experience and study objectives. Tests were usually administered in one session, in the same order as described below.

Verbal Fluency. Since processing speed and mental control are frequently affected in MS patients, three verbal fluency tasks were included in the battery. For semantic fluency, participants were asked to name as many different animals as they could [19]. Phonemic fluency was assessed using a variant of the Controlled Oral Word Association Test [20]. In our task, participants were instructed to generate as many different words as possible beginning with the letter ' $p$ '. Participants were instructed not to use proper nouns or repeat the same word with a different ending. As a new test in MS, we also asked subjects to generate as many different words as possible that did not contain the letter 'e'. Since this requires extra effort, over and above that demanded by the common task of retrieving names, this test should be particularly sensitive to cognitive dysfunction in MS patients. For the three verbal fluency tasks, each response was recorded verbatim for 60 s. Intrusions (i.e., responses different from target) and perseverations (i.e., intrusions repeated during the task) were also recorded.

Verbal Learning. The Selective Reminding Test was used to evaluate subjects' ability to remember a list of 16 unrelated words, to learn these words over three trials, and to recall these words after a 30-min delay. Subjects were first presented with cards of four words each. During the acquisition phase, subjects were asked to read the four words aloud and then say which word corresponded to different semantic categories. Free and total (i.e., free plus cued) recall, both immediately and after $30 \mathrm{~min}$, were subjected to analysis [21]. Intrusions (i.e., mistakes corresponding to the same or a different category) as well as perseverations (i.e., repeated intrusions) were recorded.

Selective Attention. A variant of the Stroop Color and Word Test was used to assess selective attention, perceptual interference, and information processing speed. The traditional test consists of reading words, naming colors, and then naming the ink color of words describing colors, where such words are printed in a nonmatching colored ink [22]. Due to time constraints, only the interference task was administered, the performance of which 
appears to be particularly impaired in MS [23]. Subjects were instructed to correct their mistakes in all cases. In addition to total correct responses in $45 \mathrm{~s}$, inhibition failures (i.e., reading the word instead of stating the color of the ink) and perseverations (i.e., a new inhibition failure following subject or examiner correction) were recorded.

Serial Additions. The Paced Auditory Serial Addition Test is a commonly used procedure in MS that combines elements of both calculation and working memory. The test requires patients to add 60 pairs of randomized digits in such a way that each is added to the immediately preceding digit. A practice task of 11 digits was given first. Digits were presented via audiotape at 2 -second intervals. The standard score for this task is the number of correct responses at each stimulus presentation rate [24]. In addition, omissions (i.e., skipped digits), interference errors (i.e., addition to the previous sum or to former digit) and other errors (e.g., calculation errors) were registered.

Visuoperceptual Ability. The Visual Object and Space Perception Battery cube analysis test was used to assess visuoperceptual ability. This task consists of determining the number of solid cubes (or 'blocks') in a picture. After two training items, the number of correct answers out of 10 was recorded [25].

Analyses

Descriptive statistics were used to summarize the demographic, clinical and cognitive characteristics of the two study groups and MS clinical subgroups. Differences between groups were analyzed using the t test, $\chi^{2}$-test and one-way analysis of variance. All tests for statistical comparisons were performed bilaterally. Redundancy between cognitive scores was investigated in the MS group using Pearson correlation coefficients. A set of cognitive scores was selected for cognitive diagnosis on the basis of sensitivity, comprehensiveness and lack of redundancy.

Two alternative approaches were explored for cognitive diagnosis: as a traditional approach, the number of failed cognitive scores was computed, and as an alternative, a global standardized (z) score was calculated for each participant. This global z score was the mean of $\mathrm{z}$ scores in the selected cognitive scores. The means and standard deviations of the control group were used to obtain $\mathrm{z}$ scores. Normality assumptions were explored in the distribution of cognitive scores, and cutoffs for abnormality were selected accordingly. The prevalence of cognitive dysfunction in MS patients was obtained by subtracting prevalence among controls from prevalence among MS patients. All statistical analyses were performed using the SPSS version 11.5 computer software program (SPSS Inc., Chicago, Ill., USA).

\section{Results}

The demographic and clinical characteristics of patients and controls are shown in table 1. Patients who participated in the cognitive study did not differ significantly from the original GEDMA cohort in terms of age, sex and clinical profile. As in the original cohort, patients suffering from progressive MS were older, had a longer disease duration and displayed a poorer functional and

Cognitive Dysfunction in MS fatigue status (comparisons between this study and the inception GEDMA cohort not shown) [12]. Patients ranged in age from 20 to 71 years (median 38), disease duration ranged from 3 to 34 years (median 12), and EDSS scores ranged from 0 to 9 (median 3.5) (table 1).

Cognitive performance for the different study groups is shown in table 2. Nine patients failed to complete one or more tests (one in the case of 7 patients, three in the case of 2 patients) owing to important cognitive impairments. Nine patients (4.7\%) were deemed to be demented. As a group, MS patients performed systematically worse than did controls. Among MS patients, those suffering from progressive disease performed worse. In general, while cognitive scores in primary progressive (PP) and secondary progressive (SP) MS were comparable, they were significantly poorer than cognitive scores registered by relapsing-remitting (RR) patients, which lay between the progressive MS and control groups. Two exceptions to this pattern should be noted: firstly, RR patients performed on a par with controls in the visual analysis task; and secondly, save for omissions in the serial additions task (which followed the general pattern described), no clear differences between MS subgroups were in evidence with respect to the type or number of errors committed (descriptive data of errors in the different tests not shown).

The performance pattern in the three verbal fluency tasks was substantially similar among MS patients and controls. As predicted, fewer correct responses were obtained in the most demanding task (i.e., 'words without e'), but this phenomenon was also observed for the normal subjects. Moreover, a similar decreasing correct-response rate pattern was observed for both MS and control subjects in the three verbal fluency tasks (responses were recorded at 15-second intervals; data not shown). Since differences between MS patients and normal subjects were most consistently observed when the total number of correct responses was used, this score was retained for the purpose of cognitive diagnosis. Correlations between the three verbal fluency scores selected were moderate, with $r$ ranging from 0.594 to 0.694 .

The verbal learning results showed that the main differences between patients and controls were in free recall. These differences became attenuated after cued recall (table 2). Memory scores were highly correlated, with Pearson coefficients ranging from 0.599 ('free recall-1', 'total recall-3') to 0.898 ('total recall-3', 'total recall, delayed') (acquisition and error scores, which offered much lower correlation coefficients, are not included here). Since maximum differences between patients and con-

Eur Neurol 736 3 
Table 1. Demographic and clinical characteristics of study groups

\begin{tabular}{|c|c|c|c|c|c|c|}
\hline & $\begin{array}{l}\text { Control } \\
(\mathrm{n}=50)\end{array}$ & $\begin{array}{l}\text { MS (total) } \\
(\mathrm{n}=191)\end{array}$ & $\begin{array}{l}\text { RR-MS } \\
(\mathrm{n}=125)\end{array}$ & $\begin{array}{l}\text { PP-MS } \\
(\mathrm{n}=14)\end{array}$ & $\begin{array}{l}\text { SP-MS } \\
(n=52)\end{array}$ & $\begin{array}{l}\mathrm{p} \text { (for MS } \\
\text { subgroups) }\end{array}$ \\
\hline Age, years & $40.2(11.7)$ & $40.1(10.5)$ & $37.5(9.3)^{\mathrm{a}, \mathrm{b}}$ & $49.1(13.8)^{\mathrm{a}}$ & $43.7(9.9)^{\mathrm{b}}$ & 0.000 \\
\hline Women, \% & 72.0 & 71.2 & 74.4 & 71.4 & 63.5 & 0.342 \\
\hline Education, \% & & & & & & 0.796 \\
\hline None & 2.0 & 3.7 & 2.4 & 7.1 & 5.8 & \\
\hline Primary & 40.0 & 39.8 & 42.4 & 35.7 & 34.6 & \\
\hline Intermediate & 36.0 & 36.1 & 36.8 & 35.7 & 34.6 & \\
\hline University & 22.0 & 20.4 & 18.4 & 21.4 & 25.0 & \\
\hline Evolution, years & NA & $12.3(6.6)$ & $11.5(6.5)^{\mathfrak{c}}$ & $12.1(6.5)$ & $14.4(6.8)^{\mathrm{c}}$ & 0.026 \\
\hline EDSS & 0 & $3.9(2.4)$ & $2.5(1.7)^{\mathrm{a}, \mathrm{a}}$ & $6.3(1.0)^{\mathrm{a}}$ & $6.4(1.0)^{\mathrm{a}}$ & 0.000 \\
\hline MMSE & NP & $26.4(2.7)(\mathrm{n}=186)$ & $26.7(2.6)$ & $26.2(2.9)$ & $25.8(3.0)$ & 0.185 \\
\hline Dementia, \% & 0 & 4.7 & 1.6 & 7.1 & 11.5 & 0.016 \\
\hline Psychiatric morbidity, \% & 0 & $44.6(\mathrm{n}=186)$ & 42.6 & 38.5 & 51.0 & 0.540 \\
\hline On interferon, $\%$ & 0 & 64.4 & 65.6 & 35.7 & 69.2 & 0.060 \\
\hline
\end{tabular}

Values are shown as mean (SD), unless otherwise indicated. ${ }^{a} \mathrm{p}<0.0005 ;{ }^{b} \mathrm{p}<0.005 ;{ }^{\mathrm{c}} \mathrm{p}<0.05$ (for differences between MS subgroups with the same superscript letter, using Scheffé's test). $\mathrm{MMMSE}=$ Mini-Mental State Examination Test; NA = not applicable; $\mathrm{NP}=$ not performed.

Table 2. Cognitive performance in the selected tasks, by study group

\begin{tabular}{|c|c|c|c|c|c|c|c|}
\hline & $\begin{array}{l}\text { Control } \\
(\mathrm{n}=50)\end{array}$ & $\begin{array}{l}\text { MS (total) } \\
(\mathrm{n}=191)\end{array}$ & $\mathrm{p}$ & $\begin{array}{l}\text { RR-MS } \\
(\mathrm{n}=125)\end{array}$ & $\begin{array}{l}\text { PP-MS } \\
(\mathrm{n}=14)\end{array}$ & $\begin{array}{l}\text { SP-MS } \\
(\mathrm{n}=52)\end{array}$ & $\mathrm{p}$ \\
\hline \multicolumn{8}{|l|}{ Verbal fluency } \\
\hline Animals & $20.1(4.6)$ & $17.1(6.3)$ & 0.000 & $18.2(6.1)^{\mathrm{d}}$ & $14.2(5.5)$ & $15.3(6.4)^{\mathrm{d}}$ & 0.003 \\
\hline Words beginning with $\mathrm{p}$ & $15.8(5.0)$ & $12.6(5.2)$ & 0.000 & $13.6(5.2)^{\mathrm{b}}$ & $11.1(3.7)$ & $10.7(5.1)^{\mathrm{b}}$ & 0.002 \\
\hline Words without $\mathrm{e}^{1}$ & $13.4(4.5)$ & $10.1(4.5)$ & 0.000 & $10.9(4.3)^{\mathrm{c}}$ & $8.1(4.4)$ & $8.6(4.5)^{\mathrm{c}}$ & 0.003 \\
\hline \multicolumn{8}{|l|}{ Verbal learning $^{2}$} \\
\hline Free recall-3 (16) & $11.0(2.6)$ & $9.2(3.1)$ & 0.000 & $9.6(2.8)$ & $8.4(3.4)$ & $8.4(3.7)$ & 0.063 \\
\hline Total recall-3 (16) & $15.1(1.6)$ & $14.1(2.6)$ & 0.002 & $14.4(2.1)$ & $14.2(3.1)$ & $13.4(3.5)$ & 0.090 \\
\hline Free recall, delayed (16) & $11.4(2.4)$ & $9.3(3.6)$ & 0.000 & $9.9(3.3)^{c}$ & $8.3(4.1)$ & $8.1(3.9)^{\mathrm{c}}$ & 0.004 \\
\hline Total recall, delayed (16) & $14.6(1.7)$ & $13.7(2.9)$ & 0.003 & $14.0(2.4)$ & $13.5(3.2)$ & $12.8(3.7)$ & 0.062 \\
\hline \multicolumn{8}{|l|}{ Selective attention ${ }^{3}$} \\
\hline Stroop, interference task & $38.9(8.5)$ & $32.6(12.0)$ & 0.000 & $35.3(11.3)^{\mathrm{a}, \mathrm{d}}$ & $26.9(12.3)^{\mathrm{d}}$ & $26.9(11.2)^{\mathrm{a}}$ & 0.000 \\
\hline \multicolumn{8}{|l|}{ Serial additions ${ }^{4}$} \\
\hline PASAT (60) & $42.0(11.8)$ & $33.9(15.5)$ & 0.000 & $36.3(14.9)^{\mathrm{d}}$ & $30.1(13.9)$ & $28.9(16.2)^{\mathrm{d}}$ & 0.010 \\
\hline \multicolumn{8}{|l|}{ Visual perception ${ }^{2}$} \\
\hline VOSP, cube analysis (10) & $9.2(1.2)$ & $9.0(1.8)$ & 0.452 & $9.3(1.3)^{\mathrm{d}, \mathrm{d}}$ & $7.9(3.5)^{\mathrm{d}}$ & $8.5(2.1)^{\mathrm{d}}$ & 0.003 \\
\hline Errors, $\mathrm{n}$ & $10.9(5.8)$ & $18.6(11.7)$ & 0.000 & $18.5(11.2)$ & $18.5(13.7)$ & $18.6(12.4)$ & 0.998 \\
\hline Global z score & $0.0(0.7)$ & $-0.7(1.0)$ & 0.000 & $-0.5(0.9)^{b}$ & $-1.1(1.2)$ & $-1.1(1.1)^{b}$ & 0.001 \\
\hline
\end{tabular}

Data are shown as mean (SD); maximum scores are shown in parentheses. The number of errors was computed as the sum of errors in verbal fluency, verbal learning and selective attention tests. ${ }^{a} \mathrm{p}<0.0005,{ }^{b} \mathrm{p}<0.005,{ }^{\mathrm{c}} \mathrm{p}<0.01,{ }^{\mathrm{d}} \mathrm{p}<0.05$ (for differences between MS subgroups with the same superscript letter, using Scheffé's test). PASAT = Paced Auditory Serial Addition Test; VOSP = Visual Object and Space Perception Battery.

${ }^{1} \mathrm{n}=190$ for MS group. ${ }^{2} \mathrm{n}=189$ for MS group. ${ }^{3} \mathrm{n}=184$ for MS group. ${ }^{4} \mathrm{n}=190$ for MS group. 
Table 3. Frequency of abnormal cognitive performance according to diagnostic approach

\begin{tabular}{|c|c|c|c|c|c|c|c|c|}
\hline & \multicolumn{4}{|c|}{ Number of failed scores ( $<5$ th p.) } & \multicolumn{4}{|c|}{ Cutoff in global z score } \\
\hline & 1 & 2 & 3 & 4 & $<5$ th p. & $<10$ th p. & $<25$ th p. & $<50$ th p. \\
\hline MS group & 56.5 & 42.4 & 27.2 & 21.5 & 14.7 & 32.5 & 52.9 & 83.2 \\
\hline Control group & 18.0 & 8.0 & 6.0 & 2.0 & 4.0 & 10.0 & 24.0 & 50.0 \\
\hline MS minus control & 38.5 & 34.4 & 21.2 & 19.5 & 10.7 & 22.5 & 28.9 & 33.2 \\
\hline
\end{tabular}

Values represent percentages of individuals; $\mathrm{n}=191$ (MS group) and 50 (control group). Percentiles and global $\mathrm{z}$ score were calculated using control group data as reference. Missing values (MS group) were treated as normal performance. $\mathrm{p}$. $=$ Percentile.

trols were obtained in free recall at the third learning trial ('free recall-3') and in free delayed recall, these two scores were retained for the purpose of cognitive diagnosis. Total recall scores (i.e., immediate and delayed total recall) were also included to reflect the fact that a considerable proportion of patients achieved normal performance once the semantic cue was given.

Patients performed significantly worse than control subjects both in serial additions and in the Stroop test, whereas visuoperceptual ability was only poorer in MS patients who presented with progressive clinical course (table 2). Correlations between serial additions, Stroop, visuoperceptual ability and the formerly selected scores were low to moderate, ranging from 0.267 ('VOSP, cube analysis', 'Stroop, interference task') to 0.590 ('Stroop, interference task', 'words without e'). Consequently, the number of correct responses in serial additions, Stroop and cube analysis were selected for cognitive diagnosis.

Patients and controls alike committed very few errors in each individual test, with the exception of omissions in the serial addition task. For this reason, Spearman's rather than Pearson's correlation coefficients were used in error analyses. Correlations between error scores were very variable, ranging from -0.138 (intrusions in 'animals', perseverations in 'delayed recall') to 0.641 (intrusions in immediate recall, intrusions in delayed recall). This variability was attributed to the low number of errors in most tests. Correlations between errors and correct responses in the same test were also very variable, ranging from -0.672 ('total recall, delayed', intrusions in delayed recall) to 0.176 ('free recall, delayed', perseverations in immediate recall). Again, this variability was attributed to the low number of errors. A lone exception to this pattern should be noted: correlation between correct additions and omissions in the serial addition test was particularly high $(\mathrm{r}=-0.887)$. On the assumption that all errors in the battery could be due to a common cognitive

Cognitive Dysfunction in MS dysfunction factor, a global error score was obtained from the sum of errors in all tests, other than serial addition omissions. Serial addition omissions were not included to avoid overweight and redundancy. This final 'global error score' was markedly altered in the MS group, was not redundant (Pearson's $r$ between the global error score and the other selected cognitive scores ranged from -0.106 to -0.508 ), and was finally added to the other 10 scores selected (table 2).

Four out of 11 selected cognitive scores ('free recall-3', 'total recall, delayed', 'VOSP, cube analysis' and 'number of errors'), as well as the global z score, displayed a skewed distribution in the control group, due to a bigger tail on the poor-performance distribution side. Hence, percentiles rather than standard deviations were chosen for determining abnormality. Cognitive dysfunction frequencies according to different cutoffs under both traditional and global z score scenarios are shown in table 3 . A better trade-off between abnormal performance in MS patients and normal performance in control subjects was obtained when the number of failed tasks, rather than the global $\mathrm{z}$ score, was used. A cutoff of two failed tasks offered the best balance, yielding a cognitive dysfunction prevalence of $34.4 \%$ (table 3 ). As for the 9 demented patients, their number of failed tasks ranged from six (1 patient) to 10 (3 patients). All demented patients had a global z score below the 5 th percentile.

The most frequently abnormal scores in MS patients were 'free recall, delayed' (27.5\%), 'number of errors' (26.2\%) and 'animals' (25.7\%), whereas the least frequently abnormal scores were 'VOSP, cube analysis' (7.9\%), 'total recall-3' (8.5\%) and 'words beginning with p' (12.0\%). The corresponding figures for the subgroup of patients registering only one or two abnormal scores were: 'number of errors' (37.5\%); 'Stroop, interference task' (32.1\%); 'animals' (26.8); 'total recall-3' (0\%); 'free recall-3' (1.8\%); and 'free recall, delayed' (1.8\%). 


\section{Discussion}

An intermediate-length neuropsychological battery was successfully administered to a clinic-based sample of 191 MS patients and 50 matched controls. Applying a 'conservative' criterion of two abnormal scores, the prevalence of cognitive dysfunction was $34 \%$, a figure that may seem slightly low when compared to previous studies. This discrepancy could be due to methodological is sues. In a recent study, a prevalence of 56\% was obtained on the basis of eight cognitive scores. However, a more 'liberal' cutoff of one altered score was chosen, and results were not corrected for abnormal performance in control subjects [26]. Remarkably, choosing only one abnormal score as cutoff and omitting control group correction would have yielded an exact correspondence, namely, a cognitive dysfunction prevalence of $56 \%$, in our study. However, using one failed score as cutoff for cognitive dysfunction might lack specificity, in the light of the $18 \%$ frequency of such an outcome in the control group (table 3). In another study, a cutoff of two altered tests was used, and $31 \%$ of patients were diagnosed as cognitively impaired. Patients were slightly less functionally $\equiv$ ired and control-group correction was not used [27].

The appropriateness of correcting cognitive dysfunction frequency with data from a control group lies in the fact that low cognitive performance may be due to several reasons other than genuine cognitive decline. Subjects could perform poorly in response to cognitive testing due to lack of motivation, lack of fam $\equiv$ ity with testing conditions, and other contingencies. Rao et al. [1], after correcting for low performance in control subjects, and using an extensive battery ( 31 cognitive scores), obtained a $43 \%$ prevalence of cognitive dysfunction in MS. However, extensive testing may not necessarily lead to a higher frequency of cognitive dysfunction if low performance in control subjects is taken into account. Indeed, more than 10 years after the seminal study by Rao et al. [1], it is earlier diagnosis and improvement in treatment that might arguably account for the decline in cognitive dysfunction prevalence in our sample. Other sources of variability, particularly those concerning setting and recruitment methodology, may also be responsible for differences in the reported frequencies of cognitive dysfunction in MS.

Rather than determining the prevalence of cognitive dysfunction in MS, our study was aimed at optimizing cognitive assessment via an intermediate-length battery. Although global standardized scores have been used in the assessment of cognitive dysfunction in MS, the authors are not aware of former studies directly contrasting the two approaches (i.e., global vs. task-specific scores). Use of the number of failed tests proved better at discriminating between patients and controls than did a single standardized global score (table 3 ). This finding is in line both with a view of MS as a heterogeneous disorder and with a modular perspective of brain functioning. A variety of pathogenic processes would be differentially represented among individual patients, giving rise to differences in both lesion topography and quality of cognitive manifestations $[28,29]$.

Rao's Brief Repeatable Battery is the most widely used battery for detecting cognitive disturbances in MS. A recent clinic-based study, using a cutoff of two altered scores and correcting for control group performance, reported a prevalence of cognitive dysfunction of $43 \%$. This higher prevalence could be attributable to our battery lacking enough demanding visuoperceptual tasks (such as visual memory and symbol-digit tasks, both present in the Brief Repeatable Battery) [30]. A limitation of our battery resides in the fact that only one relatively easy task was used to assess visuoperceptual abilities (table 2). Traditionally, visuoperceptual abilities have been reported to be less frequently altered in MS [1]. However, $6 \%$ of our minimally impaired patients failed the visuospatial task and thus the possibility of a lack of sensitivity due to visuoperceptual underrepresentation remains. The value of having more numerous and demanding visuoperceptual tasks in screening batteries for MS patients warrants further research.

By way of original contributions, we introduced a demanding verbal fluency task, reduced the Stroop test, and registered the number of errors in most tests. As a result, battery sensitivity and efficiency improved, due to error scoring in particular. Most registered errors were intrusions, perseverations and interference failures, which can be interpreted as a breakdown in mental processes dealing with temporal storage and manipulation of information, mainly depending on frontal-subcortical circuitry [31]. Future inclusion of error scores in MS cognitive batteries may increase their sensitivity at a very low cost.

In conclusion, an intermediate-length battery was adequate for cognitive screening in a large sample of MS patients, though advantages vis-à-vis other existing batteries could not be demonstrated. Once positive cases are detected, screening should be followed by a comprehensive neuropsychological assessment to better capture MS cognitive diversity and plan cognitive and functional remediation. Establishing different patterns and mechanisms of cognitive dysfunction may help approach lesion topography and understand the complex biological mechanisms of MS. 


\section{Acknowledgments}

The authors would like to thank the following: J. Casillas for her performance of the neuropsychological assessments and all the neurologists who kindly supplied patients for the study, namely, M.C. Gutiérrez del Olmo, M.A. Morales, R. Saiz, L. Ayuso, E.
Martín Zurdo, A. Miralles, P. Barreiro, E. Rodríguez, R. Arroyo, C. Martín Estefanía, C. Ramo, L. Vela, A. García Merino, J. Balseiro, C. de Andrés, M.L. Garcia de la Rocha, J.F. Plaza, J.C. Alvarez Cermeño, B. Felgueroso, and M.E. Villar. This work was supported by grants from Schering-Plough (Award in Research on Multiple Sclerosis, 1999) and Schering-España.

\section{References}

1 Rao SM, Leo GJ, Bernardin L, Unverzagt F Cognitive dysfunction in multiple sclerosis I. Frequency, patterns, and prediction. Neurology 1991;41:685-691.

2 Rao SM, Leo GJ, Ellington L, Nauertz T, Bernardin L, Unverzagt F: Cognitive dysfunction in multiple sclerosis. II. Impact on employment and social functioning. Neurology 1992;41:692-696.

3 Benedict RH, Wahlig E, Bakshi R, Fishman I, Munschauer F, Zivadinov R, et al: Predicting quality of life in multiple sclerosis: accounting for physical disability, fatigue, cognition, mood disorder, personality, and behavior change. J Neurol Sci 2005;231:29-34

4 Benedict RH: Integrating cognitive function screening and assessment into the routine care of multiple sclerosis patients. CNS Spectr 2005;10:384-391.

5 Beatty WW, Goodkin DE: Screening for cognitive impairment in multiple sclerosis: an evaluation of the Mini-Mental State Examination. Arch Neurol 1990;47:297-301.

6 Kujala P, Portin R, Ruutiainen J: Memory deficits and early cognitive deterioration in MS. Acta Neurol Scand 1996;93:329-335.

7 Beatty WW: Assessment of cognitive and psychological functions in patients with multiple sclerosis: considerations for databasing. Mult Scler 1999;5:239-243.

8 Amato MP, Ponziani G, Siracusa G, Sorbi S Cognitive dysfunction in early-onset multiple sclerosis: a reappraisal after 10 years. Arch Neurol 2001;58:1602-1606.

9 Benedict RH, Fischer JS, Archibald CJ, Arnett PA, Beatty WW, Bobholz J, et al: Minimal neuropsychological assessment of MS patients: a consensus approach. Clin Neuropsychol 2002;16:381-397.

10 Denney DR, Lynch SG, Parmenter BA, Horne N: Cognitive impairment in relapsing and primary progressive multiple sclerosis: mostly a matter of speed. J Int Neuropsychol Soc 2004; 10:948-956.
11 Litvan I, Grafman J, Vendrell P, Martínez JM: Slowed information processing in multiple sclerosis. Arch Neurol 1988;45:281285.

12 Morales-Gonzalez JM, Benito-León J, Rivera-Navarro J, Mitchell AJ; GEDMA Study Group: A systematic approach to analyse health-related quality of life in multiple sclerosis: the GEDMA study. Mult Scler 2004;10: 47-54.

13 Poser CM, Poser CM, Paty DW, Scheinberg L, McDonald WI, Davis FA, et al: New diagnostic criteria for multiple sclerosis. Ann Neurol 1983;13:227-231.

14 Kurtzke JF: Rating neurologic impairment in multiple sclerosis: an expanded disability status scale (EDSS). Neurology 1983;33: 1444-1452.

15 Cella DF, Dineen K, Arnason B, Reder A, Webster KA, Karabatsos G, et al: Validation of the functional assessment of multiple sclerosis quality of life instrument. Neurology 1996;47:129-139.

16 Folstein MF, Folstein SE, McHugh PR: Minimental state: a practical method for grading the cognitive state of patients for the clinician. J Psychiatr Res 1975;12:189-198.

17 Iriarte J, Katsamakis G, de Castro P: The Fatigue Descriptive Scale (FDS): a useful tool to evaluate fatigue in multiple sclerosis. Mult Scler 1999;5:10-16.

18 American Psychiatric Association: Diagnostic and Statistical Manual of Mental Disorders, ed 4. Washington, American Psychiatric Association, 1994.

19 Rosen WG: Verbal fluency in aging and dementia. J Clin Neuropsychol 1980;2:135146.

20 Spreen O, Benton AL: Neurosensory Center Comprehensive Examination for Aphasia. Victoria, University of Victoria Neuropsychology Laboratory, 1977.
21 Buschke H, Fuld PA: Evaluating storage, retention, and retrieval in disordered memory and learning. Neurology 1974;24:10191025.

22 Golden CJ: Stroop Color and Word Test. Chicago, Stoelting, 1978.

23 Vitkovitch M, Bishop S, Dancey C, Richards A: Stroop interference and negative priming in patients with multiple sclerosis. Neuropsychologia 2002;40:1570-1576.

24 Gronwall DM: Paced auditory serial-addition task: a measure of recovery from concussion. Percept Mot Skills 1977;44:367373.

25 Warrington EK, James M: Visual Object and Space Perception Battery. Bury St Edmunds, Thames Valley Test, 1991.

26 Savettieri G, Messina D, Andreoli V, Bonavita S, Caltagirone C, Cittadella R, et al: Sexrelated effect of clinical and genetic variables on cognitive impairment in multiple sclerosis. J Neurol 2004;251:1208-1214.

27 Filippi M, Alberoni M, Martinelli V, Sirabian G, Bressi S, Canal N, et al: Influence of clinical variables on neuropsychological performance in multiple sclerosis. Eur Neurol 1994;34:324-328.

28 Bielekova B, Kadom N, Fisher E, Jeffries N, Ohayon J, Richert N, et al: MRI as a marker for disease heterogeneity in multiple sclerosis. Neurology 2005;65:1071-1076.

29 Benedict RH, Zivadinov R, Carone DA, Weinstock-Guttman B, Gaines J, Maggiore $\mathrm{C}$, et al: Regional lobar atrophy predicts memory impairment in multiple sclerosis. Am J Neuroradiol 2005;26:1824-1831.

30 Sepulcre J, Vanotti S, Hernández R, Sandoval G, Cáceres F, Garcea O, et al: Cognitive impairment in patients with multiple sclerosis using the Brief Repeatable Battery-Neuropsychology test. Mult Scler 2006;12:187195.

31 Baddeley AD: Is working memory still working? Eur Psychologist 2002;7:85-97. 